\title{
Exceptional Cause of Hemorrhagic Shock in Intensive Care by Arterial Blood Pressure Catheter Disconnection
}

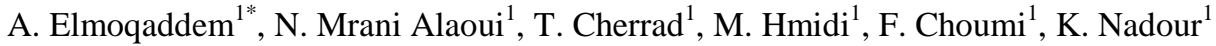 \\ ${ }^{1}$ Anesthesiology and Inten. Sive Care Department, Department of Emergency, Moulay Ismail Military Hospital, Meknes, Morocco
}

\begin{tabular}{ll}
\hline DOI: $10.36348 /$ sjmps.2021.v07i04.001 & | Received: 18.10 .2020 | Accepted: 03.11 .2020 | Published: 02.04 .2021 \\
*Corresponding author: Amine Elmoqaddem &
\end{tabular}

Abstract

The occurrence of hemorrhage from an intra-arterial catheter remains exceptional despite the risk of disconnection described for the femoral site. The occurrence of a state of hemorrhagic shock on the arterial blood pressure line still remains accidental and may be responsible for high morbidity and mortality. We report a case of hemorrhagic shock that occurred in intensive care following an accidental disconnection in the blood pressure system.

Keywords: Hemorrhagic shock; arterial catheter.

Copyright (C) 2021 The Author(s): This is an open-access article distributed under the terms of the Creative Commons Attribution 4.0 International License (CC BY-NC 4.0) which permits unrestricted use, distribution, and reproduction in any medium for non-commercial use provided the original author and source are credited.

\section{INTRODUCTION}

Postoperative hemorrhagic shock remains linked to the type of surgery and the surgical procedure, but the occurrence of a state of hemorrhagic shock on the arterial blood pressure line still remains accidental and may be responsible for high morbidity and mortality. We report a case of hemorrhagic shock that occurred in intensive care following an accidental disconnection in the blood pressure system.

\section{CASE RePORT}

This is a 50 -year-old patient with no notable pathological history admitted for a subarachnoid hemorrhage diagnosed with a brain scan, the arteriography revealed a aneurysm of the left Sylvian artery at its bifurcation. The patient was treated medically with neurological improvement giving way to restlessness.

The patient was admitted to the operative room again without signs of intracranial hypertension, the neurological examination found agitation and partial right deficit. After induction and intubation; invasive monitoring with plug of a right femoral venous line and right femoral bloody arterial line by a 18 gauge Teflon catheter, then sutured to the skin, panning opsite, after failure of radial puncture by infiltration of the upper limbs after local disinfection, palpation and auscultation of the right femoral pulse. Brain relaxation and maintenance by TCI have been insured; without particular incidents during the operation which lasted four hours. The pose of clip was performed without incident. Then the patient was transferred to intensive care unit. Extubation was performed one hour after the end of the operation, after that, the patient woke up with a GCS at 13 and without deficit; the postoperative period was marked by restlessness. The incident happened at 8:00 a.m. when the nurses team changed by disconnecting the arterial line whose catheter stays in place and squirts under the patient's sheets, after a few minutes around $2500 \mathrm{ml}$ of blood has been lost with onset of shock with blood pressure was measured at $60 /$ $25 \mathrm{mmHg}$ and a heart rate of 140 ; installation of consciousness disorders with GCS at 8 . The arterial line reconnection was performed and the patient was rapidly intubated with massive filling with macromolecules and administration of vasoactive drugs: low dose adrenaline until systolic blood pressure reaches $100 \mathrm{mmHg}$. The transfusion was performed with six CG and five PFC. Stabilization of the hemodynamic state was performed with epinephrine discontinuation, Brain CT was performed which did not show intracerebral rebleeding. The patient was extubated twenty-four hours later, deconditioned, then transferred the next day to the neurosurgery department.

\section{Discussion}

The occurrence of hemorrhage from an intraarterial catheter remains exceptional despite the risk of disconnection described for the femoral site [1]. The indications for taking a arterial blood pressure line for intraoperative monitoring invasive are cardiovascular surgery, intracranial surgery, surgery for 
pheochromocytoma, also for any surgery involving sudden variations and / or significant blood volume [1]. In our case, the indication was retained for intracranial surgery for clipping an aneurysm; the passage to the femoral site was carried out after failure of the radial site which remains the privileged site [1]. The most important complications for an arterial catheterization are shown mainly by thrombosis which may be responsible for ischemia, the incidence of which is $0.2 \%$ especially for the radial and foot sites and catheter infection [2], and less frequent fatty or cruoric emboli, arteriovenous fistulas, false aneurysms, arterial dissection, catheter migration, accidental injections intra-arterial and injection site hematoma (a). In addition, the disconnection of the catheter remains an exceptional complication [1] this was our case, an unexpected incident which risky transforming the vital prognosis of our patient. Mistakes in the placement of invasive catheters are not uncommon, are potentially serious and are always to be feared when placing any catheter, and stress the importance of to put in place very strict prevention procedures. The occurrence of hemorrhage from an intra-arterial catheter remains exceptional despite the risk of disconnection described for the femoral site.

\section{CONCLUSION}

The occurrence of hemorrhage from an intraarterial catheter remains exceptional despite the risk of disconnection described for the femoral site and may be responsible for high morbidity and mortality.

\section{REFERENCES}

1. Baron, J.F., Camus, C., Chemla D., Duranteau, R. (1995). Arterial catheterization and invasive blood pressure measurement in anesthesia- reanimation in adults. 1995; Ann Fr Anesth- reanim; 14: 444-453

2. Lambert, D., Martin, C., Perrin, G., Saux, P., Papazian L., Gouin, F. (1990). Thrombogenic risk during prolonged artery catheterization radial: comparison of two types of catheters. Ann Fr Anesthereanim, 9: 408-411. 